\title{
Covid-19: Researchers launch app to track spread of symptoms in the UK
}

\author{
Susan Mayor
}

London, UK

Nearly 700000 people signed up in the first 24 hours of the launch of an app to help track the spread of coronavirus across the UK in real time and collect information on the range of self-reported symptoms and the characteristics of those most at risk.

The Covid Symptom Tracker app (covid.joinzoe.com) enables users to make a daily report of any symptoms. It is available free of charge to members of the public in the UK. The app has also been given to 5000 twins and their families recruited from the ongoing TwinsUK cohort study. ${ }^{1}$

"The aim of the app is to help slow the outbreak" by understanding what measures work, said the researchers from King's College London and Guy's and St Thomas' Hospitals in partnership with ZOE, a spin-off health data science company from King's. "It will help to identify how fast the virus is spreading in an area, high risk areas in the country, and who is most at risk, by better understanding symptoms linked to underlying health conditions."

Lead researcher Tim Spector, professor of genetic epidemiology at King's College London, told The BMJ, "It's pretty obvious to anybody-you don't have to be an epidemiologist—-to see that we're trying to fight this war in the dark. The government doesn't have any good data on what's going to hit us in two weeks, other than what's happened in Italy and China.

"The focus has been on the number of deaths with no indication of how many people have the disease in the UK. The lack of testing means we have no clue about the prevalence in the population or about the proportions of mild and severe cases. Estimates of how many cases are symptomatic range from about $5 \%$ to $60 \%$."

People using the app are asked to provide personal information, including age, sex, height, weight, and postcode and list any chronic health conditions, including heart, lung, or kidney disease, diabetes, and regular use of immunosuppressants or non-steroidal anti-inflammatory drugs.

They are then asked about covid-19-whether they have been tested and any symptoms they are experiencing at the time, including fever, persistent cough, unusual fatigue, shortness of breath, diarrhoea, confusion, disorientation or drowsiness, and loss of appetite. They are also asked whether they have attended a hospital because of covid-19 symptoms.

People taking part in the twin study reporting symptoms will be sent a home testing kit for covid-19 to better understand what symptoms truly correspond to the coronavirus infection. Testing will help to distinguish mild covid-19 symptoms from unrelated illnesses with similar symptoms by correlating their symptom reports with test results.

Researchers will also compare genetically identical twins with non-identical twins to explore how genetic and environmental factors previously recorded in the TwinsUK cohort influence the symptoms and progression of covid-19 in different people. The researchers hope to be able to better understand why some people develop severe or fatal disease while others have only mild symptoms.

The data collected will be anonymised and made available to public health or other non-profit research institutions carrying out research on covid-19, including the NHS. "We are doing this as an open source. Every 24 hours, we'll make the data available so epidemiologists and clinical researchers can use the data in real time," said Spector. "No information shared by users will be used for commercial purposes."

The group is currently in negotiations with NHSX, which works on digital transformation, to take the app forward. It plans to launch the app in the US in the next few days.

Another app, US Health Weather Map, developed by Kinsa Insights in collaboration with Oregon State University, is being used to track "atypical illness levels" in the US by collecting data on self-reported fever. ${ }^{2}$ Although not designed specifically for covid-19, it is hoped that this may help to monitor regional distribution and trends in the transmission of coronavirus.

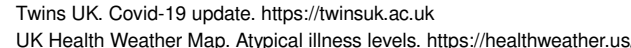

Published by the BMJ Publishing Group Limited. For permission to use (where not already granted under a licence) please go to http://group.bmj.com/group/rights-licensing/ permissions 